\title{
Electrocatalysis on Pt/YSZ electrodes
}

\author{
B. Luerßen ${ }^{\text {a }}$, J. Janek ${ }^{\text {a, } *}$, R. Imbihl ${ }^{\text {b }}$ \\ ${ }^{a}$ Physikalisch-Chemisches Institut, Justus-Liebig-Universität Gießen, Heinrich-Buff-Ring 58, D-35392 Giessen, Germany \\ ${ }^{\mathrm{b}}$ Institut für Physikalische Chemie und Elektrochemie, Universität Hannover, Callinstr. 3-3A, D-30167 Hannover, Germany
}

\begin{abstract}
Electrochemical polarization of porous metal electrodes on solid electrolytes often leads to an increase of their catalytic activity in heterogeneous reactions. Thin microstructured Pt films on single crystalline YSZ (yttria stabilized zirconia) are investigated as structurally and geometrically well-defined model systems in order to understand the origin of this effect. Photoelectron emission microscopy (PEEM) and scanning photoelectron microscopy (SPEM) have been applied as spatially resolving methods in situ to study the processes in the vicinity of the three-phase boundary (tpb). Measurements with SPEM show that atomic oxygen is created under anodic polarization and covers the Pt film homogeneously. A specific spillover species is not found, rather the formation of atomic oxygen is detected, which has the same $\mathrm{O} 1 s$ binding energy as chemisorbed oxygen from the gas phase.
\end{abstract}

Keywords: Catalysis; Electrochemistry; Interfaces; Photoelectron emission microscopy; Scanning photoelectron microscopy

\section{Introduction}

The catalytic activity of metals in a large number of heterogeneous reactions is well known. A very common example is the oxidation of carbon monoxide on a Pt catalyst. Due to extensive studies by Vayenas et al., it is now also well known that the catalytic properties of a metal can be changed by electrochemical polarization, i.e. if the metal is used as an electrode in a solid state galvanic cell. As Vayenas et al. [1] demonstrated for more than 40 different reactions, the application of such metal/ solid electrolyte electrodes may lead to a strong rate

\footnotetext{
* Corresponding author. Fax: +49-641-99-34509.

E-mail address: janek@phys.chemie.uni-giessen.de (J. Janek).
}

increase. The solid electrolyte mostly used in these studies is the oxygen ion conductor YSZ (yttrium stabilized zirconia) on which porous metal films are deposited. The yield of catalytic reactions can be increased by a factor up to 200 as shown by Vayenas, and the rate increase being observed upon application of a suitable electric potential surmounts the electric current up to a reported factor of $10^{5}$. Due to this strong non-linear relation between the ionic current (through the solid electrolyte) and the catalytic rate increase, this promoting effect is often called 'non-faradaic electrochemical modification of catalytic activity', abbreviated as 'NEMCA'.

Vayenas et al. [2] attribute this effect to an electrochemically produced spillover species formed at the three-phase boundary and spreading over the metal surface. The spillover species shall create an 
'effective double layer' and influence the catalytic behaviour. However, both the nature of the spillover species and the exact microscopic mechanism of the promotion are not yet clarified.

It is the aim of our experimental study to obtain more information by investigating a geometrically well-defined electrode model system. This electrode system consists of a microstructured thin $\mathrm{Pt}$ film deposited on YSZ single crystals being studied under chemically well-defined experimental conditions in an ultra high vacuum chamber.

In our experiments-by combining electrochemical and in situ microspectroscopic methods-we attempted to answer two essential questions: firstly, what is the chemical identity of the so-called spillover species? Secondly, can we prove that this species is formed at the three-phase boundary? In this case, we should be able to observe diffusion fronts of this species with suitable in situ methods.

\section{Theory}

A standard three-electrode set-up with the catalytically active metal as working electrode (WE) on one side of the YSZ sample and the counter (CE) and reference electrode (RE) on the other side is depicted in Fig. 1. Without applying an electric potential to this cell, an electromotive force is established, which only depends on the oxygen activities in the gas phases above the working and the reference electrode:

$V_{\mathrm{WR}}(I=0)=\frac{1}{2 F} \cdot\left[\mu_{\mathrm{o}}(\mathrm{WE}, \mathrm{gas})-\mu_{\mathrm{o}}(\mathrm{RE}, \mathrm{gas})\right]$

This arrangement is a simple concentration cell, and the observed catalytic rate shall be denoted as $r_{0}$ $(I=0)$.

The catalytic rate $r(I)$ that is observed if an anodic potential is applied to the working electrode should be higher than in the zero-current case, since oxygen as the oxidizing agent is now transported towards the surface. However, beyond this expected Faradaic rate increase, one often observes much larger effects. The factor $\Lambda$ is introduced by Vayenas to describe the measured rate increase $\Delta r=r-r_{0}$ relative to the theoretical rate increase in the Faradaic case:

$\Lambda=\frac{r(I)-r_{0}}{I / 2 F}$

$\Lambda=1$ corresponds to the Faradaic case ( $F$ being the Faradaic constant), and $\Lambda$ can take values up to $10^{5}[2]$.

A number of empirical relations have been derived by Vayenas et al. [2,10], relating $r(I)$ and $\Lambda$ to $V_{\mathrm{WR}}$ and the electrode properties. These can be interpreted on the basis of thermodynamic and ki-
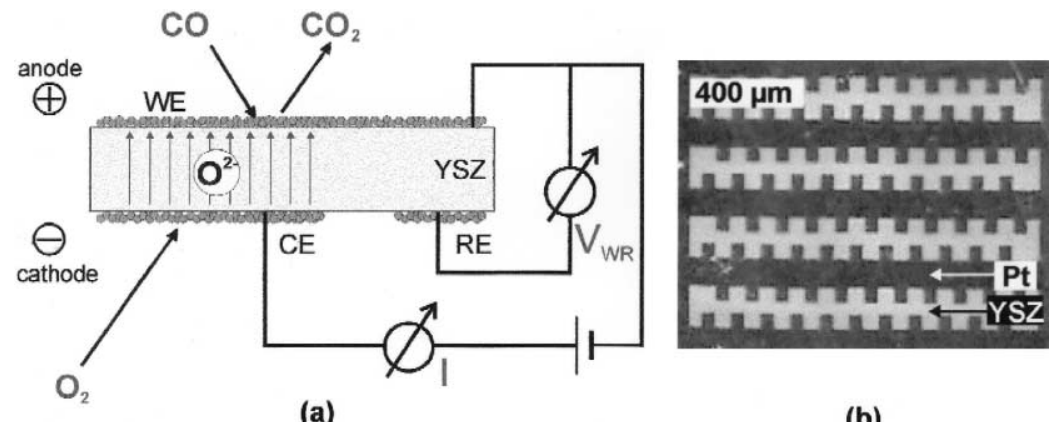

(a)

(b)

Fig. 1. (a) Schematic drawing of the electrochemical cell consisting of a YSZ single crystal ( $d=1$ mm diameter) with the working electrode on the top and the counter and reference electrode on the back side. (b) Optical micrograph of the microstructured electrode. 
netic arguments as a consequence of exponential relations between (a) the concentration of a surface species and its chemical potential, and (b) between overvoltage and current. For details, the reader is referred to Ref. [6].

\section{Experimental}

For the electrochemical measurements we used the microfabricated Pt electrode on the top side of the YSZ pellet as working electrode and a counter and a reference electrode-both prepared with $\mathrm{Pt}$ paste (Demetron Leitplatin 308 A) —on the back side of the YSZ single crystal (purchased from Kristallhandel Kelpin (Leimen, Germany)). The applied potential is controlled with a potentiostat. If the working electrode is positively charged, oxygen ions are transported towards the anode where they are discharged to create molecular oxygen.

As analytical methods, scanning photoelectron microscopy (SPEM) [3] and photoelectron emission microscopy (PEEM) [4] have been applied to study the processes at the three-phase boundary.

The SPEM measurements were performed at the storage ring Elettra at Trieste. The X-ray beam from the synchrotron is focused to a small spot of $\approx 0.15$ $\mu \mathrm{m}$ diameter by means of a zone plate optics. Two operation modes are possible: (a) spectroscopy from a small spot and (b) imaging by scanning the sample while selecting a specific kinetic energy with the analyser. During the measurements presented here, we used $643.2 \mathrm{eV}$ photons and an energy resolution of $0.5 \mathrm{eV}$ to take spectra at fixed points of the surface.

With photoelectron emission microscopy, the sample is illuminated by a $\mathrm{D}_{2}$ discharge lamp and the emitted photoelectrons are collected on a channel plate and visualized on a phosphorous screen. Therefore, low work function areas appear bright, high work function areas appear dark in the PEEM images. The spatial resolution is $\approx 5 \mu \mathrm{m}$. The work function is sensitive to changes of the surface dipole character (in this case: Pt metal and YSZ solid electrolyte), and thus is changed when a gas is adsorbed. In the case of oxygen, an increase in oxygen coverage leads to an increase of the work function of $\mathrm{Pt}$, and correspondingly, the PEEM image darkens.

The temperature was chosen between $350^{\circ} \mathrm{C}$ and $400^{\circ} \mathrm{C}$ in order to have a sufficient ionic conductivity of the solid electrolyte. All experiments were conducted under UHV conditions with a base pressure $p<10^{-9}$ mbar.

\section{Results and discussion}

The first and basic experiment is to compare by local XPS the chemical identity of oxygen, which is adsorbed on Pt from the gas phase with electrochemically generated oxygen.

Fig. 2 shows three XPS spectra [5]. Fig. 2(a) depicts the O1 $s$ signal of the Pt surface after several cleaning cycles. The remaining oxide components (Pt and Si, labelled as I1, I2, and I3) are most probably a result of the sample preparation. These components remain constant during all experiments, thus Fig. 2(a) represents the oxygen 'free' reference spectrum.

The first experiment (Fig. 2(b)) is the adsorption of oxygen from the gas phase under a constant oxygen partial pressure of $p_{\mathrm{O}_{2}}=1 \times 10^{-6}$ mbar. The well-known peak for chemisorbed oxygen at a binding energy of $530.4 \mathrm{eV}$ appears (labelled as ' $\mathrm{P} 1$ '), and the spectrum corresponds to the stationary oxygen coverage reached in adsorption/desorption equilibrium. The oxygen is turned off, the surface is cleaned again, and now in Fig. 2(c), the polarization experiment starts with an anodic potential of +1.1 $\mathrm{V}$, such that oxygen is electrochemically 'pumped' to the surface. The same peak as in Fig. 2(b) (at a binding energy $530.4 \mathrm{eV}$ ) rises up, and thus, we have to conclude that the same adsorbed oxygen species is formed.

Two small differences occur: the peak due to 'pumped' oxygen is about 1.5 times larger than in Fig. 2(b). Additionally, a second component (labelled as ' $\mathrm{P} 2$ ') is visible at a slightly lower binding energy of $530.0 \mathrm{eV}$. This second peak probably results from oxygen adsorbed on different adsorption sites, which are only populated at higher coverages. The difference in binding energies is too small to be attributed to real chemical differences or different charge states. 


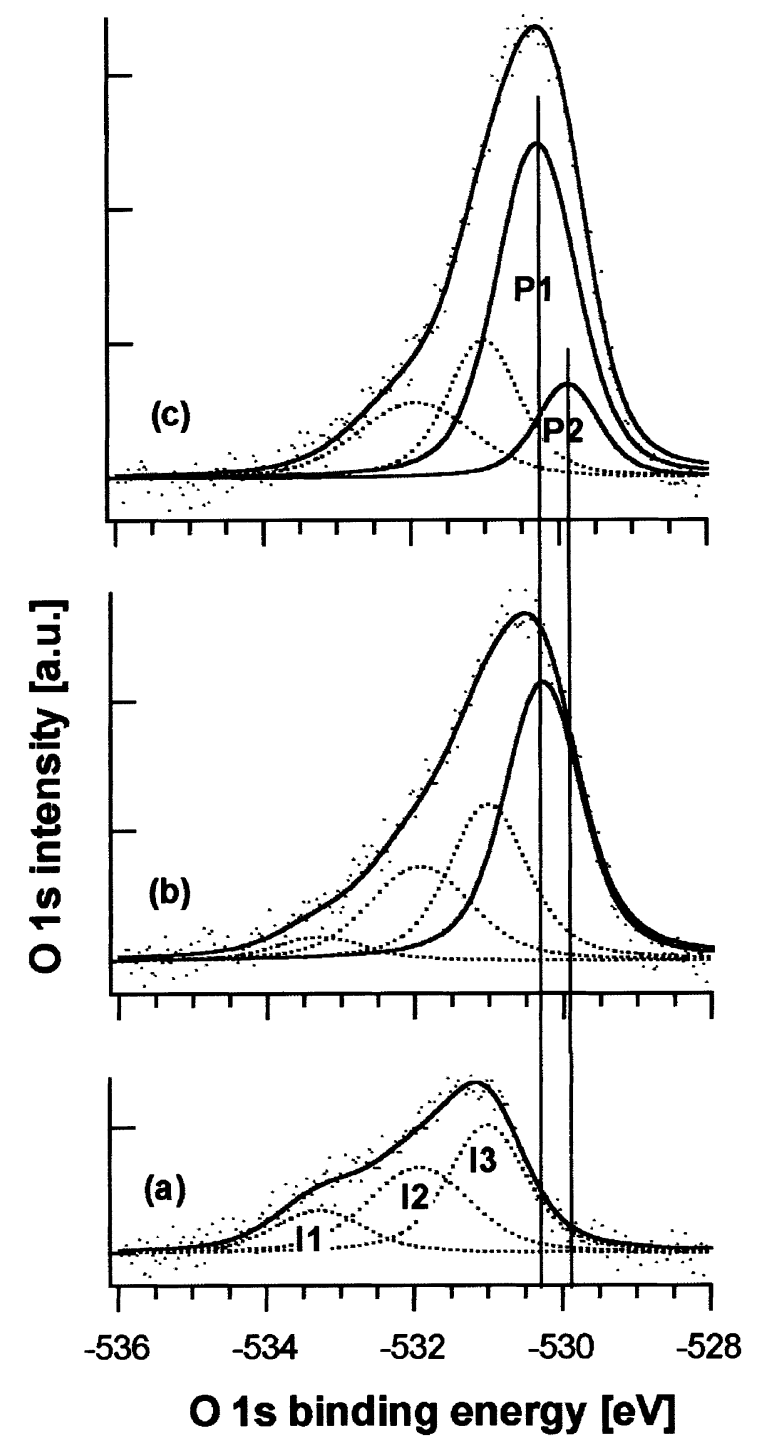

Fig. 2. Comparison between local XPS spectra of oxygen adsorbed on Pt from the gas phase and the electrochemically generated oxygen: (a) the residual $\mathrm{O} 1 \mathrm{~s}$ spectrum after the cleaning cycles; (b) the $\mathrm{O} 1 s$ spectrum measured under a constant oxygen pressure $p_{\mathrm{O}_{2}}=1 \cdot 10^{-6} \mathrm{mbar}$; and (c) the $\mathrm{O} 1 \mathrm{~s}$ spectrum during anodic polarization of the $\mathrm{WE}\left(V_{\mathrm{WR}}=+1.1 \mathrm{~V}\right)$.

This clearly leads to the conclusion that adsorbed and electrochemically generated oxygen are identical under our experimental conditions $\left(p_{\max }\left(\mathrm{O}_{2}\right) \leq 1 \times\right.$ $10^{-6}$ mbar). However, the electrochemical way of 'producing' adsorbed oxygen seems to be more effective under the given conditions.
In a second experiment, we tried to answer the question where exactly the electrochemically generated oxygen is created and how it distributes along the surface. Here, photoelectron emission microscopy was applied as a method to map the work function of the polarized Pt film.

The left picture of a series of PEEM images in Fig. 3 shows a part of the microstructured Pt surface. Bright areas represent the Pt metal, dark areas the solid electrolyte YSZ; the characteristic length of a Pt square is $\approx 40 \mu \mathrm{m}$.

Once a positive (i.e. anodic) potential is applied (in this case $+0.4 \mathrm{~V}$ ), the $\mathrm{Pt}$ areas darken-as expected-due to an increased oxygen coverage. This darkening occurs homogeneously, and within the spatial resolution of $\approx 5 \mu \mathrm{m}$ and the temporal resolution of video frames $(40 \mathrm{~ms})$, no increased oxygen concentration at the three-phase boundary indicating a diffusion front could be detected.

Taking $40 \mu \mathrm{m}$ as the characteristic diffusion length (typical length of microstructure) and $40 \mathrm{~ms}$ as temporal resolution one estimates from

$D_{i}=\frac{1}{4}\left(\frac{\bar{l}^{2}}{\tau}\right)$

a diffusivity of $D \approx 1 \cdot 10^{-4} \mathrm{~cm}^{2} / \mathrm{s}$. If the spillover species diffuses faster, any diffusion front would not be detectable in the PEEM experiment. Literature values for oxygen diffusion on $\mathrm{Pt}(110)$ are lower ( $D \approx 10^{-7} \mathrm{~cm}^{2} / \mathrm{s}$ at $T \approx 670 \mathrm{~K}[8]$ ), and thus, it should be possible to image diffusion fronts if the spillover species spreads from the tpb. A possible explanation for the lack in a diffusion front could be the porosity of the thin Pt film $(d=50 \mathrm{~nm})$, so that the characteristic diffusion length for the given time resolution gets smaller than assumed. Thus, so far our attempt to see diffusion fronts of electrochemically produced oxygen has failed.

In a third experiment, we applied a cathodic potential to the $\mathrm{Pt}$ electrode since an increase in catalytic activity is also often observed upon polarization with $V_{\mathrm{WR}}<0$ [9]. And in this case, we find also a change of the solid electrolyte during electrochemical polarization. After applying the negative (cathodic) potential to the Pt electrode (in this case: $V_{\mathrm{WR}} \approx-0.4 \mathrm{~V}$ ), a bright zone is observed in PEEM 

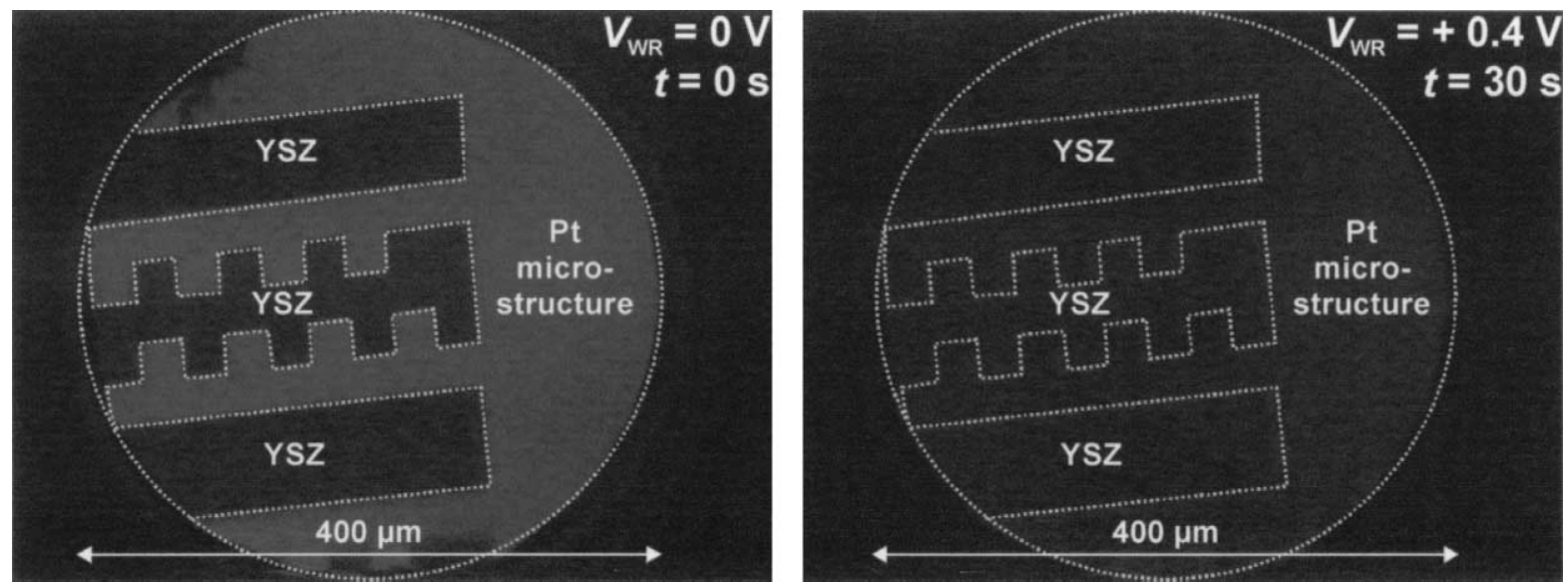

Fig. 3. PEEM images of the microstructured Pt electrode without polarization (left image) and upon anodic polarization (right image; $\left.V_{\mathrm{WR}}=+0.4 \mathrm{~V}\right)$. The dotted lines indicate the edges of the Pt electrode.

after a few seconds, which spreads on the zirconia starting from the three-phase boundary and which finally covers the complete electrolyte surface (Fig. 4). Again, this brightness change is equivalent to a

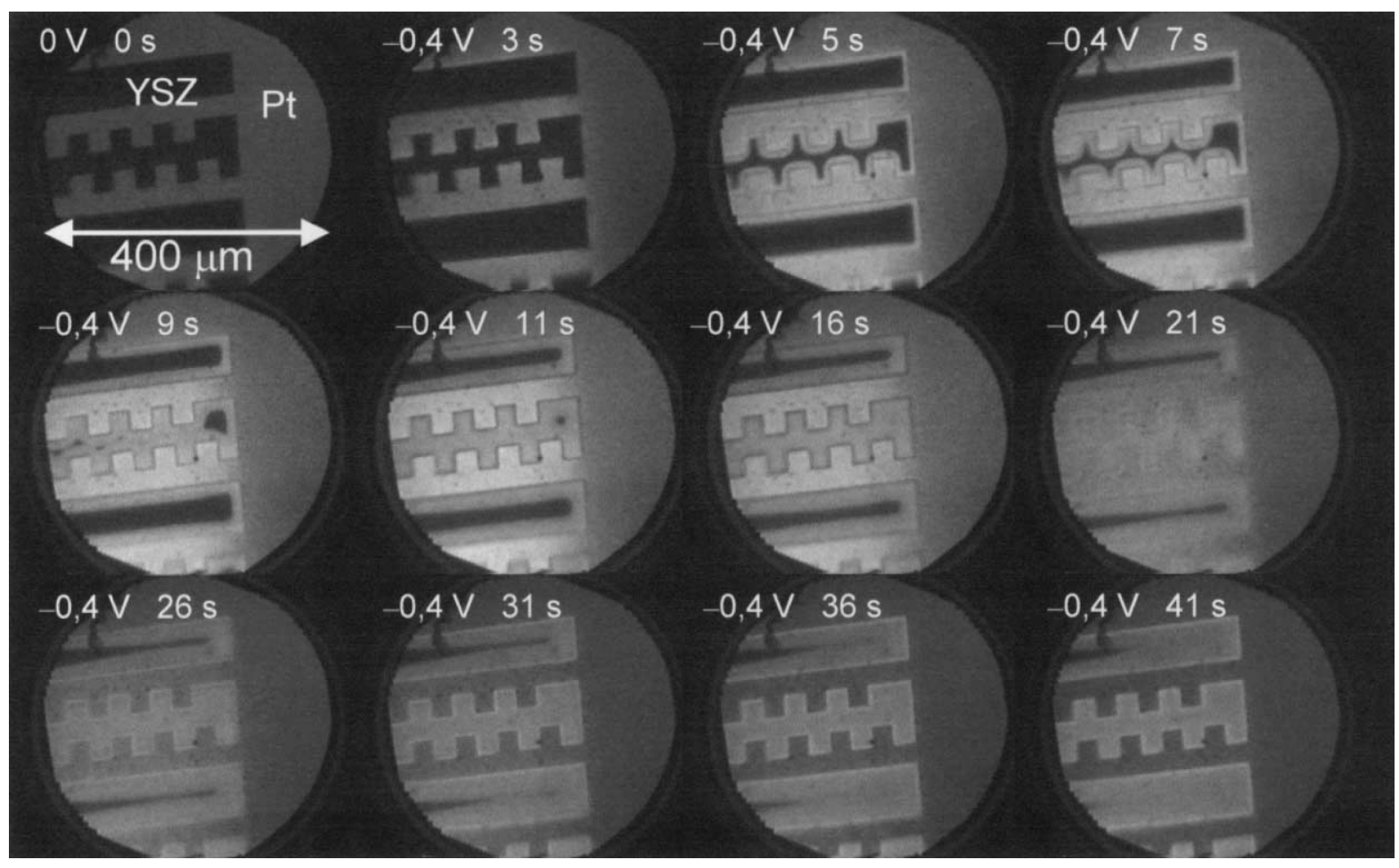

Fig. 4. PEEM images of the surface of a microstructured Pt electrode used as working electrode in a Pt/YSZ/Pt polarization cell (Fig. 1). Bright areas correspond to a low local work function, dark areas to a high work function. The series of images was taken during a polarization experiment with an anodic potential of $V_{\mathrm{WR}} \approx-0.4 \mathrm{~V}$ and shows a moving reduction front starting from the three-phase boundary, which finally covers the complete electrolyte surface. 


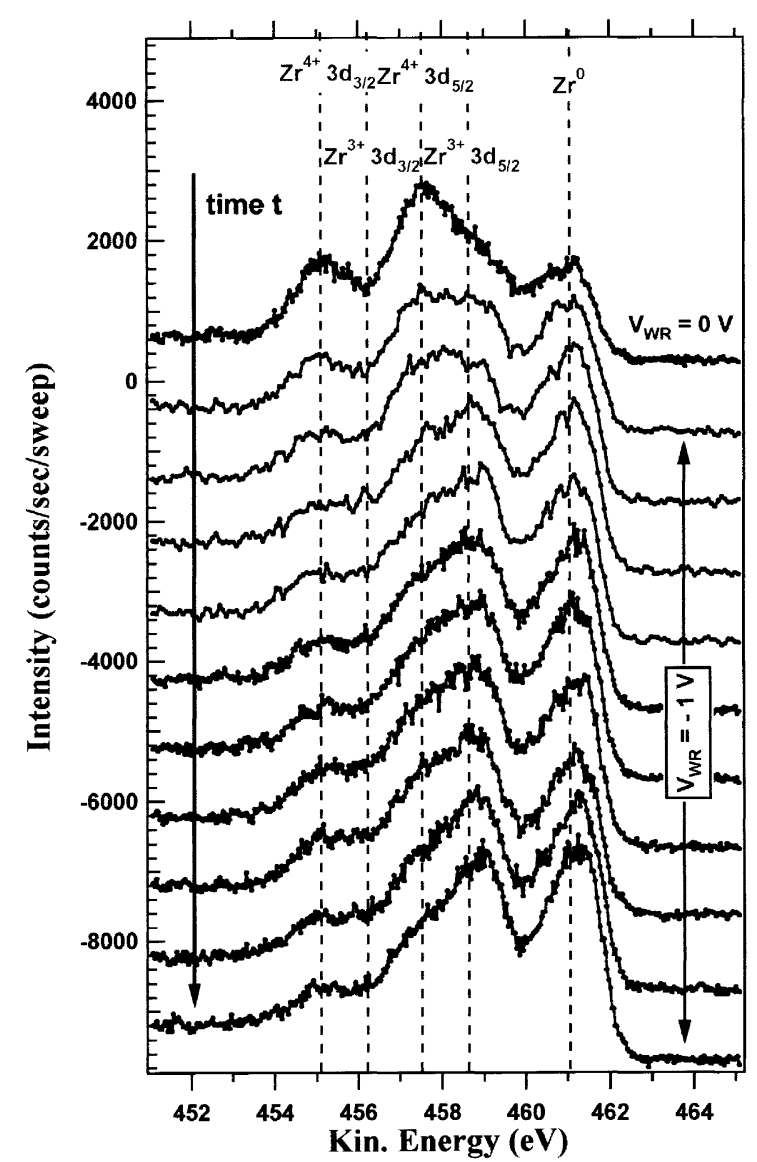

Fig. 5. Time series of XPS local Zr3 $d$ spectra of solid electrolyte YSZ surface at fixed point in a distance of $\approx 20 \mu \mathrm{m}$ to the three-phase boundary. The first spectrum on top was taken without any applied potential. A negative (cathodic) potential of $V_{\mathrm{WR}} \approx-1 \mathrm{~V}$ is adjusted and a reduction front on the YSZ starts from the tpb. The following spectra show the change of the $\mathrm{Zr}$ spectrum after the reduction front has reached the beam spot where the spectra are taken.

change of the work function. However, in contrast to metals, the work function of compounds can be modified both by changes in the electron concentration and by changes of the surface dipole. If we assume that the surface dipole remains constant, we image_-via work function changes-local changes of the electron concentration with PEEM. Thus, the brightening of the YSZ is interpreted as a local increase of the electron concentration due to the reduction of YSZ [6].

To obtain more information on the identity of the reduced electrolyte, we observed a moving reduction front again with SPEM: approximately $20 \mu \mathrm{m}$ away from the three-phase boundary, we took a series of $\mathrm{Zr} 3 d$-spectra from the zirconia surface as a function of time.

The first spectrum in Fig. 5-without any applied potential-shows the typical $3 d$-double peak of $\mathrm{Zr}^{4+}$ and already a small signal of $\mathrm{Zr}$ metal due to the reductive conditions in ultra high vacuum. Now, we applied a cathodic potential of $V_{\mathrm{WR}}=-1 \mathrm{~V}$ to the $\mathrm{Pt}$ electrode. The $\mathrm{Zr} 3 d$ spectrum indeed remained unchanged until the reduction front passes the spot where the spectra are taken. Thereafter, the contribution of reduced species-represented by peaks of $\mathrm{Zr}^{3+}$ and a growing $\mathrm{Zr}^{0}$ peak-increases; thus, it is proven that zirconia is at least partially reduced.

\section{Conclusions}

A main result of our experiments is, firstly, the observation of a spillover species at the $\mathrm{Pt} / \mathrm{YSZ}$ interface. This species has-according to SPEM measurements - a binding energy of $E_{\mathrm{B}}=530.4 \mathrm{eV}$ and is identical to chemisorbed atomic oxygen. A different oxygen species at a lower binding energy $\left(E_{\mathrm{B}}=528.8 \mathrm{eV}\right)$ found in an earlier XPS study [7] is not detected. PEEM measurements also prove the coverage with oxygen but show no enhanced oxygen concentration at the three-phase boundary or timedependant spatial intensity profiles upon anodic polarization. Since the temporal $(40 \mathrm{~ms})$ and the spatial resolution $(5 \mu \mathrm{m})$ of PEEM should be sufficient for the observation of diffusion fronts on the electrode surface, we assume that oxygen is not only created at the three-phase boundary.

Upon cathodic polarization of the Pt electrode, a moving brightness front on the solid electrolyte YSZ is observed with PEEM starting at the three-phase boundary. These low work function areas are related to a high electron concentration and reduced $\mathrm{Zr}$ species at the surface of the solid electrolyte. By SPEM measurements, the reduction of $\mathrm{ZrO}_{2}$ was confirmed.

\section{References}

[1] C.G. Vayenas, S. Bebelis, I.V. Yentekakis, H.G. Lintz, Catal. Today 11 (1992) 303.

[2] C.G. Vayenas, M.M. Jaksic, S.I. Bebelis, S.G. Neophytides, 
The Electrochemical Activation of Catalytic Reactions, in: J.O'M. Bockris et al. (Eds.), Modern Aspects of Electrochemistry, No. 29, Plenum Press, New York, 1996.

[3] L. Casalis et al., Rev. Sci. Instrum. 66 (10) (1995) 48704875.

[4] W. Engel, M.E. Kordesch, H.H. Rotermund, S. Kubala, A. von Oertzen, Ultramicroscopy 36 (1991) 148.

[5] B. Luerßen, S. Günther, H. Marbach, M. Kiskinova, J. Janek, R. Imbihl, Chem. Phys. Lett. 316 (2000) 331.
[6] J. Janek, M. Rohnke, B. Luerßen, R. Imbihl, Phys. Chem. Chem. Phys. 2 (2000) 1935-1941.

[7] S. Ladas, S. Kennou, S. Bebelis, C.G. Vayenas, J. Phys. Chem. 97 (1993) 8845.

[8] H.H. Rotermund, Surf. Sci. 283 (1993) 87-100.

[9] J. Janek, C. Korte, Solid State Ionics 116 (1999) 181-195.

[10] C.G. Vayenas, S. Bebelis, I.V. Yentekakis, Catal. Today 11 (1992) 303-442. 